\title{
Properties of the ISM in UV-luminous galaxies: clues from the low-redshift universe
}

\author{
Thiago S. Gonçalves ${ }^{1}$ \\ ${ }^{1}$ Valongo Observatory, Federal University of Rio de Janeiro, \\ Ladeira Pedro Antonio, 43, Saúde, Rio de Janeiro-RJ, CEP 20080-090, Brazil \\ email: tsg@astro.ufrj.br
}

\begin{abstract}
How is gas converted into stars across cosmic time? Observations of star-forming galaxies at high redshift indicate that the conditions of the interstellar medium (ISM) were remarkably distinct from typical spirals in the local universe. Nevertheless, these observations are biased towards objects brighter than $\mathrm{L}^{*}$, due to the large luminosity distances involved. Here I present a survey targeting the molecular gas in galaxies at low redshift $(z \sim 0.2)$ with ISM conditions remarkably similar to those observed at earlier epochs, including high star formation rates and lower metallicities. CO observations performed with CARMA indicate that these galaxies follow the same star-formation law as local spirals and other galaxies at the same redshift, albeit at much higher densities. We also present recent results from our ALMA program studying galaxies down to $12+\log (\mathrm{O} / \mathrm{H}) \sim 8$, and discuss the implications of these data to our understanding of the molecular gas reservoir and the conversion factor between CO luminosity and gas mass in environments that are simultaneously low in metal content and extremely dense.
\end{abstract}

Keywords. galaxies:ISM, galaxies:high-redshift, galaxies:starburst

\section{Introduction}

Star-forming galaxies at high redshift are remarkably different from local spirals, and are responsible for over half of the stellar mass in the universe today. However, since they are so distant, observations represent a considerable challenge, given the faint observed magnitudes. In order to alleviate the problem, we have observed a sample of UV-luminous galaxies at $z \sim 0.2$ with properties (star formation rates, morphology, metallicity, dust attenuation, gas kinematics) unlike objects with the same stellar mass at low redshift, but similar to typical $\mathrm{L}^{*}$ objects at $z \sim 2$ (Hoopes et al. 2007, Overzier et al. 2010, Gonçalves et al. 2010), which justifies their denomination as Lyman break analogs, or LBAs. This allows us to study their interstellar medium in great detail and high sensitivity, in an attempt to understand the physical processes governing star formation in under such conditions.

\section{Results}

High gas fractions: We have begun a survey searching for CO emission in LBAs, as a proxy for total gas mass. In Gonçalves et al. (2014) we have seen that LBAs have higher gas fractions (up to $67 \%$ ) than local star-forming galaxies with similar stellar masses (typically less than 10\%; Fig. 1, left). Nevertheless, these objects still lie on the local relation between star formation rates and gas reservoirs - the Schmidt-Kennicutt (SK) relation - albeit at much higher densities. This indicates that the star formation laws are universal; the challenge then is to understand how these galaxies are able to accumulate such high gas masses at very high density.

$\mathrm{CO}$ emission at low metallicities: More recently, we have used ALMA to search for CO emission in the most metal-poor objects in our sample (Gonçalves et al.2015, in prep). Although it has been determined that $\mathrm{CO}$ molecules are dissociated in such cases, one would expect the dense and turbulent ISM to counterbalance this effect (e.g., Narayanan et al.2012). Nevertheless, we do not detect $\mathrm{CO}$ in any of the 4 objects targeted, effectively meaning that these galaxies are deficient in $\mathrm{CO}$ emission given their far-infrared emission (Fig. 1, right). This has important 

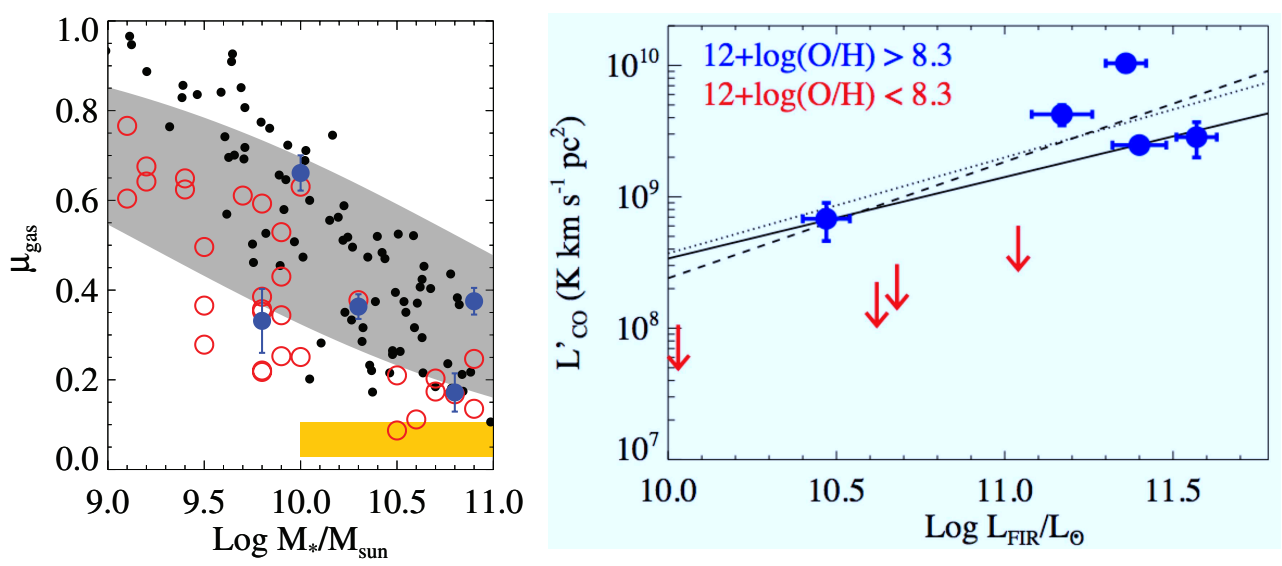

Figure 1. Left: Gas fractions of LBAs, measured with CARMA (blue) and inferred from star formation rates (red). Values are similar to those found in $z \sim 2$ (black dots, grey area), and much higher than in local SFGs (yellow area). Right: $L_{\mathrm{CO}}^{\prime}-L_{\mathrm{FIR}}$ relation for observed LBAs, with CARMA (high metallicity, in blue) and ALMA (low metallicity, in red). Low metallicity objects are undetected, with upper limits for $L_{\mathrm{CO}}^{\prime}$ well below expected values given their far infrared luminosities - solid, dotted and dashed lines.

implications regarding the detectability of $\mathrm{CO}$ emission in typical star-forming galaxies at high redshift, due to the lower metallicity determined for these objects at earlier times (Maiolino et al.2008).

\section{References}

Gonçalves, T. S., Basu-Zych, A., Overzier, R. A., et al. 2010, ApJ, 724, 1373

Gonçalves, T. S., Basu-Zych, A., Overzier, R. A., Perez, L., Martin, D. C. 2014, MNRAS, 442, 1429

Hoopes, C. G., Heckman, T. M., Salim, S., et al. 2007, ApJS, 173, 441

Maiolino, R., Nagao, T., Grazian, A. 2008, A\&A, 468, 463

Narayanan, D., Krumholz, M. R., Ostriker, E. C., Hernquist, L. 2012, MNRAS, 421, 3127

Overzier, R. A., Heckman, T. M., Schiminovich, D., Basu-Zych, A. R., Gonçalves, T. S., Martin, D. C., Rich, R. M. 2010, ApJ, 710, 979 